\section{Ethics of providing clinically assisted nutrition and hydration: Current issues}

The provision of clinically assisted nutrition and hydration $(\mathrm{CANH})$ can present clinicians with significant practical and ethical dilemmas. In this issue Andrew Rochford summarises the ethical and legal issues and offers a framework dealing with how to best support. This is in the context of an increasing elderly population and large and vulnerable patient groups including conditions such as stroke, dementia, advanced malignancy, cerebral palsy and eating disorders. The review discusses the indications, relative contraindications and alternative methods of support when CANH is not felt appropriate. The authors include four key learning points - the ethics of feeding is complicated and challenging, the first question should be 'what are we trying to achieve', CANH can be appropriately given on a trial basis and a multidisciplinary approach is essential. These issues are all dealt with comprehensively and pragmatically and the article is really helpful as a toolkit to help manage these patients. The article includes a comprehensive discussion of consent (including the legal issues), factors encouraging CANH, a useful table of questions to support decision making and discussion of exit strategies including withdrawal of treatment. Well worth working through (see page 128).

\section{Predicting the development of psychological morbidity in inflammatory bowel disease: a systematic review}

We all recognise that psychological morbidity is common in inflammatory bowel disease although factors which predict this are less clear. In this issue Hoogkamer and colleagues review the literature. There are very few studies. In their systematic review they looked 660 papers 7 of which met their inclusion criteria - all measured depression, 3 also measured anxiety. From their data risk factors for psychological morbidity included aggressive disease HR 5.77, 95\% CI 1.89-17.7, co morbidity burden (one or more additional disorders or diseases) HR 4.31, 95\% CI 2.38-6.57. Psychological risk factors included lower levels of gratitude - gratitude being defined as 'a life orientation toward noticing the positive in life, including both thankfulness to others and a wider sense of appreciation for what one has - at baseline and in adolescents with IBD high levels of parenting stress across four domains including communication, medical care, role functioning and emotional functioning. The data is interesting. There is however the need for more studies and more data and further research would enable earlier intervention for cases at high risk with the intent to reduce psychological morbidity long term (see page 137).

\section{Inflammatory bowel disease psychological support pilot reduces inflammatory bowel disease symptoms and improves psychological well-being}

In a linked article in this edition Eccles and colleagues report, in a small cohort, improved symptoms and psychological well-being following psychological intervention. Although a small study the data is powerful. Psychological services are limited. Most of us would support increased access to psychological services to improve disease outcomes and gather further evidence of efficacy (see page 154).

\section{The gut microbiome: what every gastroenterologist needs} to know

The term 'microbiome' has only been used for 20 years. It is something that we, as gastroenterologists and hepatologists need to know about. In this issue Mullish and colleagues provide an excellent update discussing the basic principles (including terminology), practical implications and future directions - diagnostic and therapeutic - in this rapidly evolving field. It is difficult to even conceptualise 100 trillion microbial cells (the microbiota) which make up the gut microbiome. The authors in their overview discuss technology like next generation sequencing of bacterial genomes using samples from multiple sites which has enhanced our 'in depth' knowledge and applied bioinformatics used to describe particular microbial patterns with the functionality being explored using other systems biology techniques including metabonomics and proteomics. The authors helpfully walk us through the multiple different techniques and terminologies. The gut microbiome is not a bystander and has multiple different benefits to the host with alteration of composition (or dysbiosis) having potential impact on health and disease. The authors discuss specifics conditions including inflammatory bowel disease, liver disease and clostridium difficile infection and the gut microbiome as therapy including pre biotics, probiotics and faecal microbiota transplant. It is great to have all the information in one place and such a comprehensive overview. Essential reading and editor's choice this month (see page 118).

\section{Guideline review: Tofacitinib for adults with moderately to severely active ulcerative colitis - NICE guidance}

Tofacitinib is an oral, Janus Kinase (JAK) molecule, which selectively inhibits Janus associate tyrosine kinase JAK1 and JAK3. In this issue Nwaogu and colleagues discuss the NICE guidance - recommended for moderate to severe active ulcerative colitis when conventional therapy or a biological agent cannot be tolerated or the disease has responded inadequately or lost response to treatment. There is good evidence of efficacy with a recommended induction then maintenance regimen. Higher doses can be used. There is however evidence of an increased risk of venous thromboembolism on higher doses and this needs to be considered. The guideline review is a useful update on a new therapeutic option - including a figure which summarises the mechanism of action - with an excellent commentary which considers the practicalities of using it in clinical practice (see page 133).

\section{How to manage postcholecystectomy abdominal pain}

The how to manage series is to give practical (and pragmatic) advice for specific clinical scenarios. In this issue Bekkali and colleagues discuss the important issue of postcholecystectomy abdominal pain - to put this in context cholecystectomy is the most frequently performed surgical operation in the UK 
- 66660 annually and up to 40\% will have pain post cholecystectomy. The authors discuss aetiology, assessment, investigation and management including discussion of the Rome IV diagnostic criteria for biliary pain and conditions like anterior cutaneous nerve entrapment syndrome, bile acid malabsorption and sphincter of oddi dysfunction. The article is clear and focused and will be very helpful for clinicians managing patients with postcholecystectomy pain (see page 145).

The how to manage series is there to help clinicians in practice. Please get in touch if you feel there are other 'how to manage' scenarios you think are particularly challenging and you think we should cover.

Please enjoy this issue. Please continue to read, enjoy and feedback on the journal.
Follow us on twitter@FrontGastro_BMJ and listen to our regular podcasts accessed via the journal website https://fg.bmj.com/

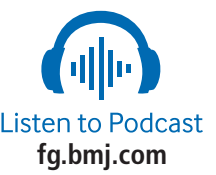

